Abstract PS2-11

Lessons Learned in Using Electronic Health Records in Management of Cardiovascular Disease

Nirav R. Shah, MD, MPH, Geisinger Center for Health Research; Zahra S. Daar, MS, Geisinger Center for Health Research; James Walker, MD, Geisinger Center for Health Research; Walter F. Stewart, $\mathrm{PhD}, \mathrm{MPH}$, Geisinger Center for Health Research

Background: The risk of cardiovascular events is reduced through the optimal management of serum lipids, blood pressure, smoking, weight, physical activity and other established risk factors. To bridge the gap between knowledge and practice, we tested an electronic health record (EHR)-based prototype care model to routinely screen for and manage cardiovascular disease (CVD) risk. Methods: We developed and tested automated protocols to accomplish six tasks: (1) identifying patients who should be evaluated for risk of CVD using a modified Framingham Risk Score at the point of care; (2) ordering appropriate tests leveraging routine physician visits; (3) determining 5-year risk of heart attack; (4) communicating risks to patients via automated letters from physicians; (5) engaging those at moderate to high risk of heart attack to modify behavior; and (6) providing physicians with clinical decision support (CDS). Results: The prototype care model was evaluated on 346 patients. Steps 1 through 4 were successfully implemented. Step 5, engaging patients in behavior change, was too limited and did not provide the patient with sufficient control over various options. Step 6, providing CDS, presented physicians with 1 of 43 fixed order sets. A more dynamic and flexible order process was needed to satisfy physician needs. Conclusions: This prototype care model presents a successful first step toward automating screening and basic care of chronic diseases while focusing care on a patient-centered model. The problems revealed through this system were largely process challenges.

\section{Abstract PS2-12 \\ Developing a Scalable Chronic Care Model for Primary Care Management of Cardiovascular Disease Risk}

Walter F. Stewart, PhD, MPH, Geisinger Center for Health Research; Annemarie G. Hirsch, MPH, Geisinger Center for Health Research; Nirav R. Shah, MD, MPH, Geisinger Center for Health Research; Ilene G. Ladd, MS, Geisinger Center for Health Research; James B. Jones, MBA, Geisinger Center for Health Research

The existing models for chronic disease management (DM), including the chronic care model (CCM) and DM offer a well-accepted conceptual framework for continuous care of patients with chronic diseases. However, there are logistical and process challenges to developing scalable primary care models consistent with these concepts. We are developing a second generation prototype care process for primary-care-based management of cardiovascular disease (CVD) that is consistent with these models. We will describe the research and development and prototyping process for creating new health services models. We will specifically describe workflow and technology solutions for the following steps in the care process: (1) identify patients who should be evaluated for CVD risk (i.e., males $45+$, females $55+$ ) using an automated and systematic process; (2) collect CVD risk data in an automatic and systematic fashion during routine primary care visits, including collection of behavioral health data via a web-based questionnaire; (3) calculate a modified Framingham Risk Score based on self-reported data (i.e. smoking, alcohol) and clinical and lab values from the medical record; (4) communicate to patients their 10-year risk of heart attack; (5) engage moderate to high-risk patients to return for a primary care visit during which they initially decide on interventions (i.e., medications, coaching, education, etc.) for each risk factor; and (6) develop sophisticated clinical decision support that completes a real-time evaluation of patient data and manufactures a draft order set, clinical notes, and after visit summary in advance of the patient and physician encounter. Finally, we will describe outcomes relevant to evaluating the prototype and plans for system-level scaling.
Abstract PS2-13

Are Diabetes Patients With Depression More Likely to Get Routine Care Process Measures?

Heidi L. Ekstrom, HealthPartners Research Foundation; Thomas J. Flottemesch, HealthPartners Research Foundation; Patrick J. O'Connor, HealthPartners Research Foundation; A. Lauren Crain, HealthPartners Research Foundation; William A. Rush, HealthPartners Research Foundation; John C. Kluznik, HealthPartners Research Foundation; Ann M. Hanson, HealthPartners Research Foundation

Background: This study was designed to test the hypothesis that diabetes patients with a depression diagnosis are less likely than those without a depression diagnosis to receive recommended frequencies of glycated hemoglobin (A1c) and lipid testing. Methods: We identified 14,144 adults with diabetes receiving care from a single large multispecialty medical group in Minnesota between 1997 and 2003. Those with two or more depression ICD-9 codes, or with one depression ICD-9 code plus at least one prescription fill for depression medication were classified as having depression. A total of 2179 met these criteria. Subjects were tracked for 4 years. Data on A1c and lipid tests were extracted from claims data and verified by chart audit in a sub-sample of patients. Results: The 2179 individuals with at least one depression episode had a mean age of 60 and had 8.2 primary care visits, on average. Eight hundred thirty-five $(38 \%)$ of these individuals were male, $1886(86 \%)$ took an antidepressant, $570(26 \%)$ had at least one other comorbid condition, $642(29 \%)$ disenrolled in the plan prior to 20 months, and $259(12 \%)$ were newly diagnosed cases of diabetes. Relative to those without a depression diagnosis, those with a depression diagnosis had significantly fewer $(P<0.001)$ than recommended A1c and low density lipoprotein (LDL) tests in bivariate analysis (30\% compared with 35\% among non-depressed cases). This finding persisted after adjustment in multivariate models for age, sex, comorbidity, number of primary care visits, and duration of the diabetes episode. Conclusions: Episodic depression is associated with sub-optimal monitoring of A1c and LDL levels in diabetes patients. These findings may help direct efforts to improve diabetes care in those with depression. They also suggest accountability measures used to assess quality of diabetes care may need to be adjusted for differences in depression occurrence across providers, clinics, or medical groups.

\section{Abstract PS2-15 \\ Diabetes as Risk Factor for Herpes Zoster Infection: \\ Results of a Population-Based Study in Israel}

Anthony D. Heymann, BSc, MB, BS, MRCGP, MHA, Maccabi Healthcare Services, Sackler Faculty of Medicine, Tel Aviv University, Israel; Gabriel Chodick, PhD, Maccabi Healthcare Services, Sackler Faculty of Medicine, Tel Aviv University, Israel; Thomas Karpati, MD, Maccabi Healthcare Services, Israel; L. Kamer, MD, Maccabi Healthcare Services, Israel; E. Kremer, MD, Maccabi Healthcare Services, Israel; Manfred D. Green, MD, Sackler Faculty of Medicine, Tel Aviv University, Israel; Ehud Kokia, MD, Maccabi Healthcare Services, Israel; Varda Shalev, MD, Maccabi Healthcare Services, Israel

Background: Studies showed that diabetes mellitus (DM) is often accompanied by impaired cell-mediated immunity, which potentially may increase the risk for infectious diseases, including herpes zoster (HZ). However, data on the relation between DM and $\mathrm{HZ}$ are scarce. This case-control study explored the association between DM and HZ. Methods: This study was nested within a cohort of all members of a large health maintenance organization (HMO) in Israel. Cases totaled 22,294 members who were diagnosed with HZ between 2002 and 2006. Controls $(n=88,895)$ were randomly selected from the remaining HMO population using frequencymatched age, sex, and duration of follow-up. Personal data on history of DM, lymphoma, leukemia, or AIDS, were obtained from computerized medical records. Results: Adjusted analyses showed that the risk of $\mathrm{HZ}$ was associated with history of leukemia, lymphoma, use of steroids or antineoplastic medications and AIDS, particularly among patients under 45 years. In a multivariate analysis, DM was associated with an increased risk of HZ (OR=1.53; 95\% CI: 1.44-1.62). Conclusions: The data suggest that individuals with DM are at increased risk of HZ. Well-designed cohort studies may help to clarify the nature of this association. 